TM-1421

0401.000

\title{
MICROWAVE INSTABILITY LIMITS WHEN DRIVEN BY NARROW RESONANCES *
}

\author{
K.-Y. Ng \\ September 1986
}

*Submitted to the Proceedings of the 1986 Summer Study on the Physics of the Superconducting Super Collider, Snowmass, Colorado, June 23-July 11, 1986 
Fermi National Accelerator Laboratory*, Batavia, IL 60510

\section{ABSTRACT}

$\left[\mathrm{Z}_{\mathrm{L}} / \mathrm{Q}\right]_{\mathrm{sh}}$ or $\left[\mathrm{Z}_{\mathrm{T}} / \mathrm{Q}\right]_{\mathrm{sh}}$ should be quoted instead of $\mathrm{Z}_{\mathrm{L}} / \mathrm{n}$ or $\mathrm{Z}_{\mathrm{T}}$ when the impedance is a narrow resonance.

\section{INTRODUCTION}

The longitudinal microwave instability formula for a Gaussian bunch is 1

$$
\left|\frac{Z_{L}}{n}\right| \leq \frac{2 \pi|\eta|(E / e)}{\beta^{2} I_{p}}\left[\frac{\sigma E}{E}\right)^{2},
$$

where $\mathrm{Z}_{\mathrm{L}} / \mathrm{n}$ is the longitudinal impedance driving the instability, $\eta$ the frequency dispersion parameter, $E$ the energy of the particle with charge $e, \sigma_{\mathrm{E}}$ its RUS spread, and $\beta$ the velocity in unit of that of light. This criterion is correct only when the impedance is a broad band wider than the frequency spectrum of the particle bunch. However, there are contributions to $\mathrm{Z}_{\mathrm{L}}$ that are sharp resonances. These sharp resonances have been proved experimentaliy able to drive microwave instabilities also. If these resonances are from cavities containing bellows or beam monitors, one likes to argue that the actual size of each module is generally slightly different so that the resonant frequency will be slightly different for each module. The sum of the resonances of these module may become a broad resonance; so broad that Eq. (1) can be applied. However, most of the time, we do not know how much the spread in the size of the module is. Also, in many cases, the total widening of the resonance is not bigger than the frequency of the bunch so that Eq. (1) cannot be applied.

Here, we give a simple intuitive derivation of a stability criterion for sharp resonances; the more exact derivation is given in the APPENDIX.

\section{STPLB DRRTYATION}

A closer look at the derivation of the criterion reveals that $\mathrm{z}_{\mathrm{L}}$ in Eq. (1) is in fact an effective impedance $\left[Z_{L}\right]_{\text {eff }}$ which is given approximately by

$$
\left[\mathrm{Z}_{L}\right]_{\text {eff }} \sim \int \mathrm{Z}_{L}\left(\mathrm{n} w_{0}+w\right) \rho_{0}(w) \mathrm{d} w,
$$

where $\omega_{0}$ is the revolution angular frequency, $n$ the harmonic where $Z_{L}$ is peaking, and $\rho_{O}(w)$ is the frequency spectrum of the unperturbed bunch which is normalized to unity. Thus, if $\mathrm{Z}_{\mathrm{L}}$ is much broader than $\rho_{\mathrm{O}}(\omega)$,

$$
\left[Z_{L}\right]_{\text {eff }} \sim Z_{L} \int \rho_{O}(w) d w=Z_{L},
$$

which is indeed the peak of the broad band and therefore we have the stability condition in Eq. (1).

If $\mathrm{Z}_{\mathrm{L}}$ is a sharp resonance at $w_{\mathrm{R}}=$ nwo $_{0}$ narrower than $\rho_{0}(w)$, we get

$$
\left[\mathrm{Z}_{\mathrm{L}}\right]_{\text {eff }} \sim\left(\frac{\sigma^{T}}{\sqrt{2 \pi}}\right)\left(\frac{\pi \omega_{\mathrm{R}}\left[\mathrm{Z}_{\mathrm{L}}\right]_{\mathrm{sh}}}{2 Q}\right),
$$

where the first bracket is the peak of the frequency

* Operated by the Universities Research Association, under contract with the U.S. Department of Energy. spectrum of the bunch with a RUS time length of $\sigma_{\tau}$ wile the second bracket is the sres under the resonant impedance. Here, $\left[\mathrm{Z}_{\mathrm{L}}\right]_{\mathrm{gh}}$ is the shunt impedance and $\mathrm{A}$ the quality factor. Substituting into Eq. (1), we obtain

$$
\left(\frac{Z_{L}}{q}\right)_{s h} \leq \frac{4|\eta|(E / e)}{\beta^{2} I_{A V}}\left(\frac{E}{E}\right)^{2},
$$

where $I_{A V}$ is the average current which is related to the peak current $I_{p}$ by

$$
\frac{I_{A V}}{I_{p}}=\frac{\omega_{0} \sigma T}{\sqrt{2 \pi}} .
$$

For narrow resonance, we think the criterion (6) is better than criterion (1) with $\mathrm{Z}_{\mathrm{L}}$ replaced by $\left[\mathrm{Z}_{\mathrm{L}}\right]_{\text {eff. }}$. The reasons are:

(a) If we quote the threshold [ $\mathrm{Z}_{\mathrm{L}}$ ] eff computed using Eq. (2) and use criterion (1), we are quoting something that is dependent on the bunch length $\sigma_{\tau}$ [see Eq. (4)] which may not be a constant during acceleration and storage.

(b) With criterion (6), we do not need to assume some sort of resonance broadening. Remember that criterion (1) can only be used when there is a broadening mechanism that makes the resonance wider than $p_{O}(w)$.

(c) $\left[\mathrm{Z}_{\mathrm{L}} / Q\right]_{\text {sh }}$ is independent of resistivity of the wall material. As a result, it can be read off readily from a URMFL computation, from TBCI computation with Fourier transform, or from a wire measurement experiment which certainly is not able to produce the correct $Q$.

For the dipole mode, by exactly the same reason, the criterion to microwave stability is

$$
\left|\mathrm{Z}_{\mathrm{T}}\right| \leq \frac{4 \sqrt{2 \pi}|\eta| \beta(\mathrm{E} / \mathrm{e}) \mathrm{n}}{\beta \mathrm{I}_{\mathrm{p}}}\left[\frac{\mathrm{E}}{\mathrm{E}}\right),
$$

Where $\hat{\rho}$ is the average betatron function, is good only when the transverse impedance $Z_{T}$ is a broad band. In the case of a narrow resonance, we have similarly,

$$
\left[\frac{\mathrm{Z}}{\mathrm{Z}}\right]_{\mathrm{sh}} \leq \frac{8 \sqrt{2} / \pi|\eta| \beta(\mathrm{E} / \mathrm{e})}{\beta \mathrm{I}_{\mathrm{AV}}}\left(\frac{\sigma_{\mathrm{E}}}{\mathrm{E}}\right) .
$$

Note that in Eq. (7), there is the harmonic number $n$ which is usually taken hand-waivingly as the cutoff harmonic or ring radius divided by beam pipe radius. But there is no such $\mathbf{n}$ in Eq. (8). This provides another reason why for a narrow resonance we should use criterion (8) rather than try some phony way to broaden the resonance and guess at an $n$ so that criterion (7) can be used.

The derivation of criteria (6) and (8) is not exact. As is carried out in the APPENDIX, the narrow resonance is approximated by a $\delta$-function with the imaginary part neglected. But the neglect of the imaginary part violates causality. However, we believe that Eqs. (6) and (8) should be correct due to intuitive foeling, although some more work needs to be done in the exact derivation. Nevertheless, we strongly advocate the quoting of two stability limits in each 


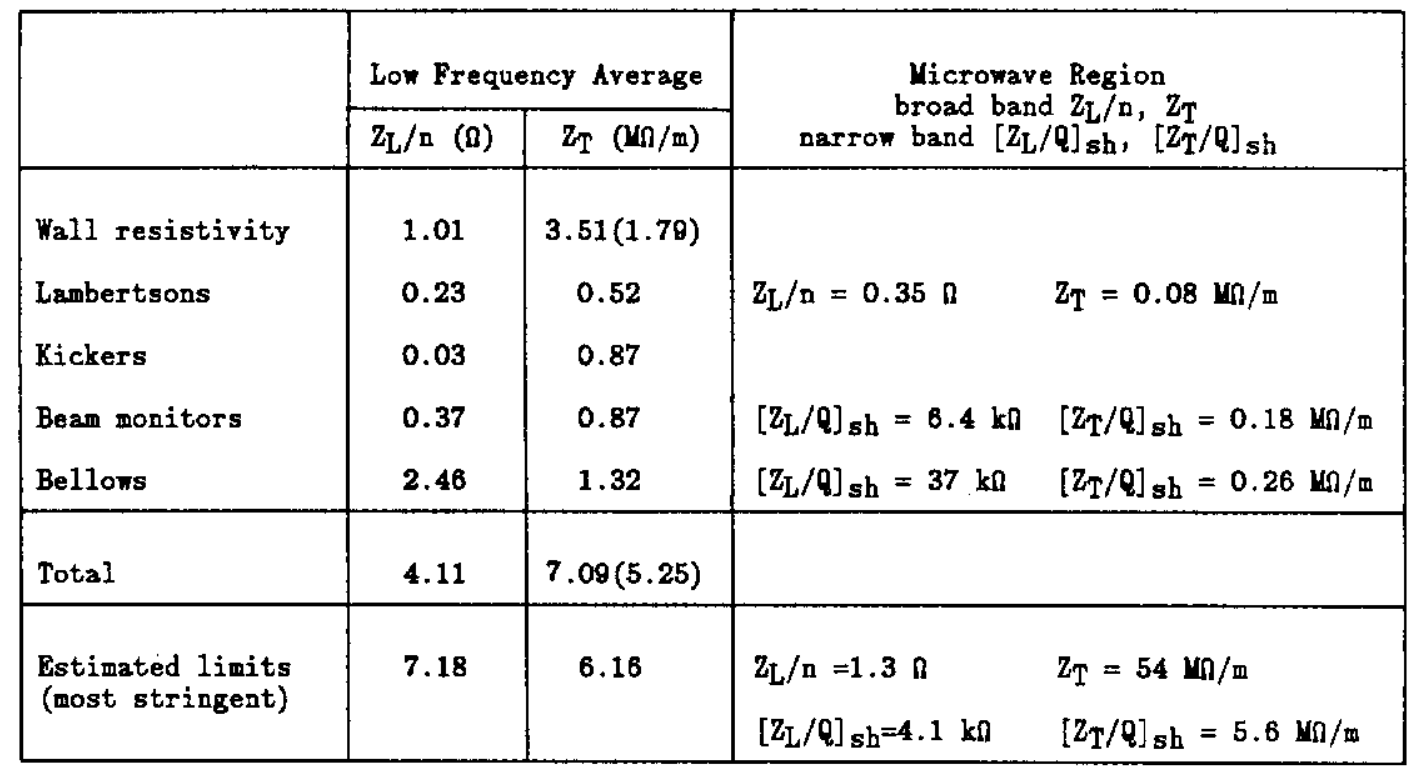

Table I. Impedance estimates and stability criteria for the Fermilab Main Ring, Whenever the vertical and horizontal transverse impedances are not the same, the vertical one is enclosed in brackets.

case, one for the broad-band $\mathrm{Z}_{\mathrm{L}}$ (or $\mathrm{Z}_{\mathrm{T}}$ ) and one for the narrow band $\left[\mathrm{Z}_{\mathrm{L}} / \mathrm{Q}\right]_{\text {sh }}$ (or $\left[\mathrm{Z}_{\mathrm{T}} / \mathrm{Q}\right]_{\mathrm{sh}}$ ). In the computation of impedances, also $\left[\mathrm{Z}_{\mathrm{L}} / \mathrm{Q}\right]_{\mathrm{sh}}$ and $\left[\mathrm{Z}_{\mathrm{T}} / \mathrm{Q}\right]_{\text {sh }}$ should be quoted. An example is shown below in Table I for the Fermilab Main Ring 3 .

\section{APPBNDIX}

According to Ref. 1 , in order that a bunch will execute microwave instability with a collective frequency $n$, the perturbed frequency density $\rho_{1}(\omega)$ must satisfy the eigen-equation ${ }^{4}$

$$
\rho_{1}\left(w^{\prime}\right)=\int \mathrm{d} \omega^{\prime} \mathrm{T}\left(w^{\prime}, w^{\prime}\right) \rho_{1}\left(w^{\prime}\right),
$$

where the kernel $T\left(w, w^{\prime}\right)$ is

$$
T\left(\omega, w^{\prime}\right)=\frac{-i \beta^{2} e I_{A V}}{\sqrt{2 \pi|\eta| \sigma_{\tau} E}}\left(\frac{E}{\sigma_{E}}\right)^{2}\left(\frac{Z_{L}\left(w^{\prime}+\Omega\right)}{w^{\prime}}\right) h(x) \rho_{0}\left(w-w^{\prime}\right) .
$$

In above,

$$
P_{\mathrm{o}}(\omega)=\frac{\sigma_{\tau}}{\sqrt{2 \pi}} \exp \left[-\omega^{2} \sigma_{\tau}^{2} / 2\right]
$$

is the same spectral distribution of the unperturbed Gaussian bunch that appears in Eq. (2) and is normalized to unity. (This $\rho_{0}$ is proportional to the function in Ref. 1.) The function $h(x)$ is defined as

$$
h(x)=\int_{0}^{\infty} \xi \mathrm{e}^{i x \xi} \mathrm{e}^{-\xi^{2} / 2} \mathrm{~d} \xi,
$$

the argument is $x=Q E /\left(|\eta| \sqrt{w w} \sigma_{E}\right)$. This function has the property that, for Ior $(x) 20$ or a growth,

$$
|\mathrm{h}(\mathrm{x})| \leq 1 \text {, }
$$

with the equality holding for $x=0$.

For a broad-band impedance centered at $\omega_{1}=n w_{0}$ much bigger than $w_{0}$ and the frequency spread of the bunch, we can substitute for $w \sim w^{\prime} \sim w_{1}$ in $Z_{L}\left(w^{\prime}+\Omega\right) / w^{\prime}$ and $h(x)$. Then, the eigen-equation ( $\theta)$ can be solved by letting $\rho_{1}(\omega)=1$, the eigenvalue gives

$$
\frac{-i \beta^{2} I_{A V}}{2 \pi|\eta|(E / e) w_{0}}\left(\frac{E}{\sigma_{\tau}}\right)^{2}\left(\frac{Z_{L}}{n}\right) h(x)=1
$$

Putting in the absolute value signs and noting Eqs. (6) and (13), we arrive at the criterion (1) for a broad band impedance.

If the impedance is a narrow resonance at $w_{R}$ with width wuch narrower than the bunch spectrum, we write

$$
Z_{L}(w) \sim \frac{\pi w_{R}}{2}\left[\frac{Z_{L}}{Q}\right]_{s h} \delta\left(\omega-w_{R}\right)
$$

Then, the eigen-equation becomes

$\rho_{1}(\omega)=\frac{-i e \beta^{2} I_{A V}}{2 \sqrt{2 \pi}|\eta| \sigma_{\tau} E}\left[\frac{Z_{L}}{Q}\right]_{s h} \rho_{0}\left(\omega-w_{R}\right) h\left(\frac{Q E}{|\eta| \sqrt{\omega \omega} \sigma_{E}}\right) \rho_{1}\left(w_{R}\right)$

Setting $\omega=\omega_{R}$, and noting that $|h(x)| \leq 1$ when there is a growth, we immediately arrive at criterion (5). However, Bq. (15) is not exact, a narron resonance should also contain an imaginary part

$$
\operatorname{Im}\left[Z_{L}(w)\right]=P \frac{w_{R}}{2 Q\left(w-w_{B}\right)},
$$

where $P$ denotes principal value. With the inclusion of the imaginary part Eq. (16) will no longer be the eigen-solution. This problem will be examined elsewhere.

\section{RBFBRBNCBS}

(1) S. Krinsky and J. M. Wang, Particle Accelerators 17, 109 (1885)

(2) J. Griffin and J. Maclachlan, IEEE Proc. Nuc1. Sci. NS-32, 2359 (1985).

(3) K. Y. Ng, Fermilab Report TM-1388 (1986).

(4) Here, we are repeating essentially what is done in Ref. 1, but we let the frequency be continuous instead of discrete so that the quality factor $Q$ will appear. 